\title{
Per-oral endoscopic myotomy for achalasia cardia: outcomes in over 400 consecutive patients
}

\section{(๑) $\circledast \ominus$}

\author{
Authors \\ Nageshwar Reddy ${ }^{1}$ \\ Institutions \\ 1 Asian Institute of Gastroenterology - Gastroenterology, \\ Hyderabad, India \\ 2 Asian Institute of Gastroenterology - Anaestheisa, \\ Hyderabad, India \\ 3 Asian Institute of Gastroenterology - Surgical \\ Gastroenterology, Hyderabad, India
}

Zaheer Nabi ${ }^{1}$, Mohan Ramchandani ${ }^{1}$, Radhika Chavan ${ }^{1}$, Rakesh Kalapala ${ }^{1}$, Santosh Darisetty ${ }^{2}$, Guduru Venkat Rao ${ }^{3}$,

submitted 25.9.2016

accepted after revision 17.1 .2017

Bibliography

DOI https://doi.org/10.1055/s-0043-105517 |

Endoscopy International Open 2017; 05: E331-E339

(c) Georg Thieme Verlag KG Stuttgart · New York

ISSN 2364-3722

Corresponding author

Zaheer Nabi, Asian Institute of Gastroenterology -

Gastroenterology, Hyderabad, India

Fax: 91-40-23324255

zaheernabi1978@gmail.com

\section{ABSTRACT}

Background and study aims Per-oral endoscopic myotomy (POEM) has emerged as an efficacious treatment modality for the management of achalasia cardia (AC) and nonachalasia spastic esophageal motility disorders. Initial results are encouraging. We analyzed the safety and efficacy of POEM in a large cohort of patients with AC.
Patients and methods The data from patients who underwent POEM (from January 2013 to June 2016) was prospectively collected and analyzed. Clinical success was defined as Eckardt score $\leq 3$ after POEM procedure. Objective parameters including high-resolution manometry (HRM) and timed barium swallow (TBS) were analyzed and compared before and after the procedure. Gastroesophageal reflux was analyzed using 24-hour pH impedance study and esophagogastro-duodenoscopy.

Results A total of 408 patients (mean age 40 years, range 4-77 years) underwent POEM during the specified period. POEM could be successfully completed in 396 (97\%) patients. Clinical success rates at 1,2 and 3 years were $94 \%, 91 \%$ and $90 \%$, respectively. Mean Eckardt score was $7.07 \pm 1.6$ prior to POEM and $1.27 \pm 1.06$ after POEM $(P=$ $0.001)$ at 1 year. Significant improvement in esophageal emptying on TBE (>50\%) was documented in $93.8 \%$ patients who completed 1-year follow up. Pre-procedure and post-procedure mean lower esophageal sphincter pressure was $45 \pm 16.5 \mathrm{mmHg}$ and $15.6 \pm 6.1 \mathrm{mmHg}$, respectively $(P=0.001)$. Technical and clinical success were comparable in naïve vs prior treated cases (97.3\% vs $96.8 \%$, $P=0.795)$ (95.7\% vs $92.6 \%, P=0.275)$. GERD was documented in $28.3 \%$ patients with 24 -hour $\mathrm{pH}$-impedance study and erosive esophagitis was seen in $18.5 \%$ of patients who underwent POEM.

Conclusions POEM is safe, effective and has a durable response in patients with achalasia cardia. Prior treatment does not influence the outcomes of POEM.

\section{Introduction}

Achalasia cardia (AC) is the most common primary motility disorder of the esophagus, characterized by absent esophageal peristalsis along with incomplete relaxation of lower esophageal sphincter (LES). The causative mechanism of AC is not well known. However, genetic predisposition along with autoimmunity to some viral agent (e.g herpes virus) may be responsible for the degeneration of myenteric neurons [1]. No currently available treatment options result in regeneration of myenteric neurons to bring back esophageal motility. However, lowering LES pressure can significantly reduce symptoms and improve quality of life. To achieve this outcome (LES pressure reduction), therapeutic techniques have been modified from time to time. Graded pneumatic balloon dilatation (PBD) has replaced conventional one-time dilatation and open Heller's myotomy has cleared the way for laparoscopic Heller's myotomy (LHM) with fundoplication.

Young patients ( $<40$ years) and those with spastic achalasia (type III AC) do not respond as well to PBD [2,3]. Moreover, repeated dilatations may be required in these patients. LHM is efficacious with durable response $[4,5]$. However, it is invasive 
with definite morbidity. Per-oral endoscopic myotomy (POEM) has emerged as a minimally invasive endoscopic treatment modality with excellent short-term results [6-8]. However, the experience is limited when compared to PBD or LHM. Long-term efficacy and incidence of gastroesophageal reflux disease (GERD) after POEM have not been well studied.

In this study we analyzed medium-term outcomes with POEM in a large cohort of patients with AC at a single tertiary care center.

\section{Patients and methods}

In this study we analyzed the prospectively collected data on all patients who underwent POEM at our center. All eligible patients presenting with AC were offered POEM from January 2013 to March 2016.

Exclusion criteria included patients unfit for general anesthesia, presence of esophageal varices, oral anti-coagulation and very young children ( $<4$ years or $<15 \mathrm{~kg}$ weight).

Diagnosis and sub-typing of AC were established using highresolution manometry (HRM). Esophagoduodenoscopy (EGD) and timed barium swallow (TBS) were done prior to POEM in all patients. In the presence of esophageal candidiasis, oral antifungals were given for 7 to 10 days prior to POEM. Symptom severity was recorded using Eckardt score which has scores for dysphagia, regurgitation, chest pain, and weight loss (range 0 to 12 ).

All intraoperative events including procedure time and complications were recorded prospectively. All patients were kept nil per oral for 1 day after the procedure. Thin barium esophagogram was done on the second postoperative day to look for any leak. Patients were started on liquid diet on Day 2 and on a soft diet from Day 3 onward.

All patients were evaluated for symptoms (Eckardt score) at pre-specified intervals: 6 months, 1 year, 2 years, and 3 years after the procedure. Objective parameters including TBS, HRM and EGD were recorded in addition to symptom assessment at the 1-year visit.

Objective assessment of GERD was done by 24-hour $\mathrm{pH}$-impedance at 3 months. Patients were asked to stop any PPI medications 5 days before the proposed date of the test. On the test day calibrated $\mathrm{pH}$ probe was placed transnasally, which was connected to a $\mathrm{pH}$ data acquisition device ( $\mathrm{ZepHr} \mathrm{pH}$ monitor with ComforTEC disposable catheters, Sandhill Scientific, Highlands Ranch, CO, USA). The $\mathrm{pH}$ measurements were recorded for 24 hours. Acid reflux episode was defined as a $\mathrm{pH}$ fall of less than 4. Twenty-four-hour esophageal acid exposure of $<4.2 \%$ was considered normal. Of all the parameters recorded in the report, total number of reflux episodes (normal<73) and composite DeMeester Score were taken into account for evaluation of GERD. DeMeester score $>14.7$ was considered as indicative of GERD.

Clinical success was defined as an Eckardt score $\leq 3$ after POEM. In cases with persistent symptoms or relapse of symptoms (Eckardt score $>3$ ), additional treatment was offered including PBD or botulinum toxin injection (BTI) or re-POEM.
Adverse events (AE) were defined as those requiring a specific intervention such as insufflation-related events requiring drainage or transiently withholding the procedure and those leading to prolonged hospitalization. Along with clinical assessment, end tidal $\mathrm{CO}_{2}$ and fluoroscopy were used to decide whether the POEM procedure should be temporarily withheld. Fluoroscopy was also used in cases with significant abdominal distension and rise in end tidal $\mathrm{CO}_{2}(>45 \mathrm{mmHg})$. The procedure was temporarily withheld in cases of retroperitoneal air or high end-tidal $\mathrm{CO}_{2}(>50 \mathrm{mmHg})$. Capnoperitoneum associated with significant abdominal distension was drained using a standard intravenous cannula.

\section{Technique}

All patients with non-sigmoid AC were kept on a liquid diet for 1 day prior to the procedure. In patients with sigmoid AC, only clear liquids were allowed for 2 to 3 days prior to the procedure. Intravenous antibiotics were started on the day of the procedure and continued for 2 days thereafter. Subsequently patients were switched to oral antibiotics for up to 7 days.

The POEM procedure was carried out under general anesthesia with patient in supine position. An upper gastrointestinal endoscope equipped with water jet (Olympus GIF HQ 190; Olympus Corp., Tokyo, Japan) was used for the procedure. A transparent cap with tapered end (DH-28GR; Fujifilm, Tokyo, Japan) was used in the initial 250 cases. In subsequent cases a standard transparent cap from Olympus was used in addition to the tapered cap. The selection of site and length of myotomy (anterior at 2 o'clock position vs posterior at 5 o'clock position) was left to the endoscopist's (DNR, ZA and MR) discretion. However, patients with spastic esophageal disorders (i.e Type III AC, jackhammer esophagus and distal esophageal spasm) were subjected to longer myotomies. A posterior approach (5 o'clock position) was used in patients who had undergone LHM previously.

The POEM procedure was carried out using the following steps-

Step 1. Submucosal injection was given using a solution of saline mixed with indigo-carmine dye to raise a wheal.

Step 2. A small incision (a few millimeters long) was made with a needle knife and enlarged vertically up to $\sim 2 \mathrm{~cm}$ with an insulated tip knife (KD-611L; Olympus, Tokyo, Japan) (๖ Fig. 1a, b).

Step 3. Subsequently, entry into the tunnel was facilitated by clearing submucosal fibers along the edges and leading apex of mucosal incision using a triangular tip knife (Triangle Tip Knife, KD-640 L, Olympus, Japan).

Step 4. A submucosal tunnel was created by dissecting the fibers using spray coagulation with a triangular tip knife ( $>$ Fig.2ab). Intervening vessels were coagulated with a coagrasper (Coagrasper G, FD-412LR, Olympus, Japan) in soft coagulation mode ( $80 \mathrm{~W}$, effect 5) ( $\mathbf{F i g} . \mathbf{3 a b}$ ).

Step 5. The tunnel was extended across the gastroesophageal junction (GEJ) for at least $2 \mathrm{~cm}$. Extension across the GEJ was confirmed by visualizing blanched gastric mucosa, characteristic vascular pattern, aberrant longitudinal muscle fiber 


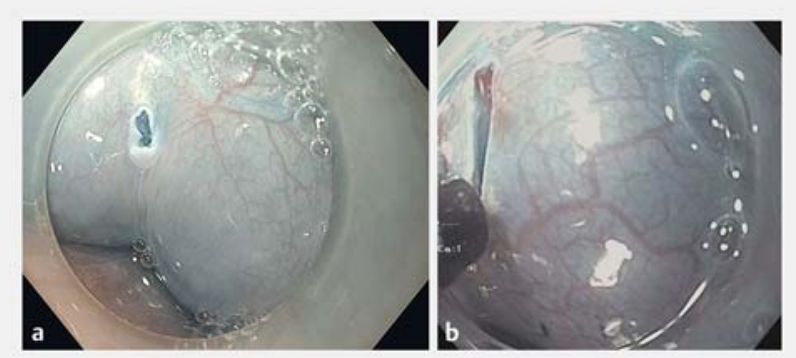

- Fig. 1 a Small mucosal incision made with a needle knife. b Extension of mucosal incision with an insulated tip knife
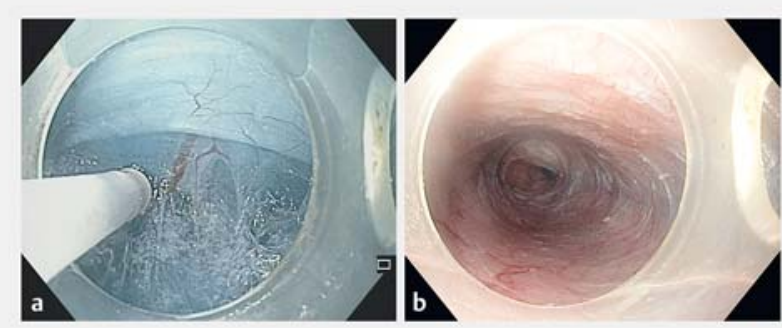

- Fig. 2 a Submucosal tunneling with use of a triangular tip knife. b Completion of submucosal tunneling.
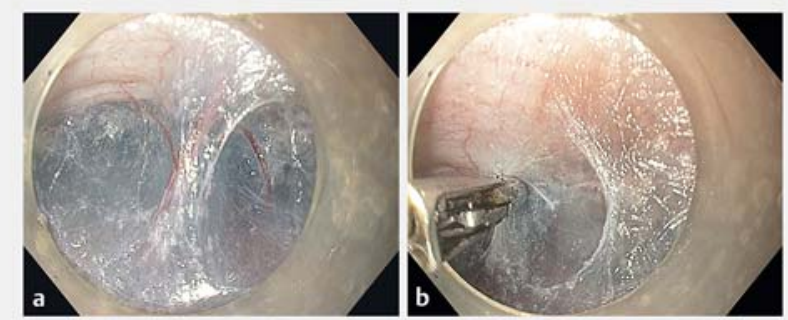

- Fig. 3 a Perforator vessel encountered during submucosal tunneling. $\mathbf{b}$ Coagulation of the same vessel with Coagrasper.

and/or the position of the tip of endoscope fluoroscopically $(\triangleright$ Fig.4a-d).

Step 6. Circular-only myotomy was done in the proximal esophagus and full-thickness myotomy was carried out from 2 to $3 \mathrm{~cm}$ above the GEJ up to the gastric end of the tunnel ( Fig. 5a,b).

Step 7. After the completion of myotomy, the esophageal and gastric mucosa were carefully inspected for any inadvertent injury. Any mucosotomy was closed with endoclips.

Step 8. The mucosal incision was closed using endoclips (EZ Clip, HX-610-090 L, Olympus, Japan) in distal to proximal fashion .

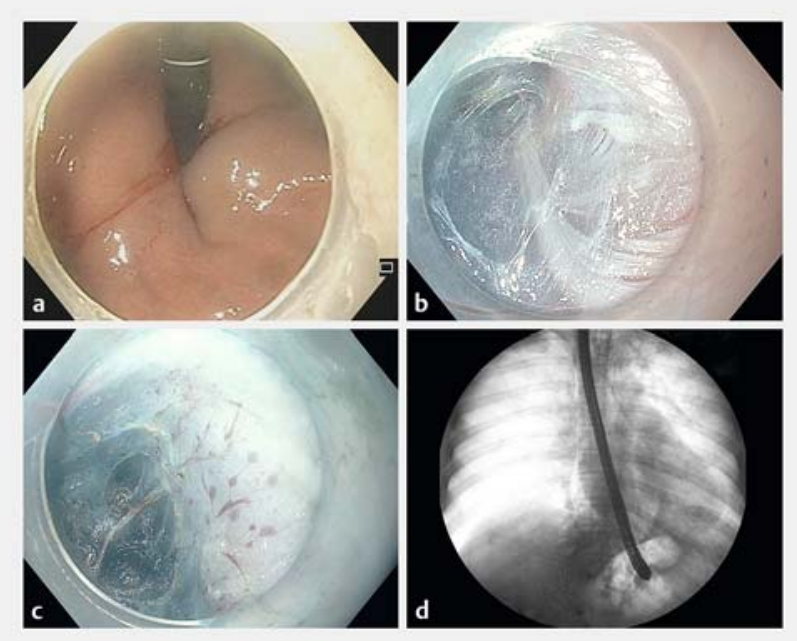

- Fig. 4 Gastroesophageal junction (GEJ) markers. a Blanched gastric mucosa suggestive of extension across GEJ. b Aberrant longitudinal muscle fibers indicating the proximity of GEJ. c Short spindle vessels on the gastric end of the submucosal tunnel. $\mathbf{d}$ Visualisation of the gastroscope under fluoroscopy to confirm extension beyond the GEJ.

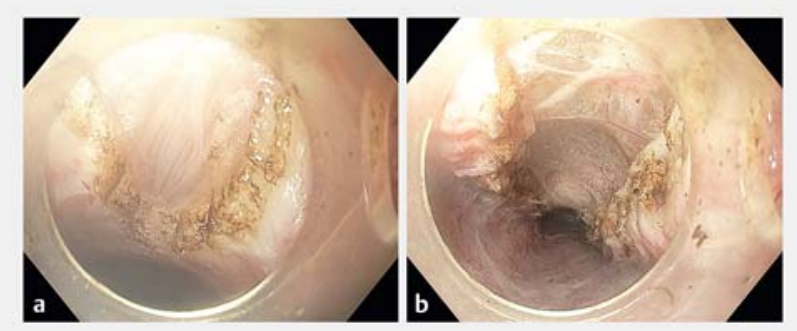

- Fig. 5 Myotomy with a triangular tip knife. a Longitudinal muscle fibers visible after selective circular myotomy. b Full-thickness myotomy at the lower end of the tunnel.

\section{Statistics}

The data were analyzed and compared before and after the POEM procedure. The data are presented as median (range) or mean \pm standard deviation. Student's paired $t$-test was used for continuous variables and proportion test for categorical variables. A $P$ value $<0.05$ was considered statistically significant.

\section{Results}

\section{Patient characteristics}

A total of 423 patients underwent POEM in our department during the study period ( $\vee$ Table 1 ). Of them, 15 patients who were diagnosed with non-achalasia spastic esophageal disorders including Jackhammer esophagus and distal esophageal spasm were excluded from the final analysis. Four hundred and eight patients (mean age 40 years, range $4-77$ years, 231 men) were classified as AC according to Chicago classification-type I $(n=128)$, type II $(n=259)$ and type III $(n=20)$. Prior treatment 


\begin{tabular}{|l|c|}
\hline Table 1 Demographics of study patients. & \\
\hline No. of patients & 423 \\
\hline Mean age, years (+SD,range) & $40 \pm 13.8(4-77)$ \\
\hline Male: female & $231: 177$ \\
\hline Disease duration (months) & $18.6(3-106)$ \\
\hline Indication of POEM & \\
\hline - Achalasia cardia & 408 \\
\hline - Type I & 128 \\
\hline - Type II & 259 \\
\hline - Type III & 20 \\
\hline - Distal esophageal spasm & 9 \\
\hline - Jack Hammer esophagus & 6 \\
\hline Previous therapy & \\
\hline - Botulinum injection & 3 \\
\hline - Pneumatic balloon dilatation (PBD) & 162 \\
\hline - Heller's Myotomy (LHM) & 19 \\
\hline - Combined (PBD and LHM) & 5 \\
\hline - POEM & 3 \\
\hline POEM-peroral endoscopic myotomy & \\
\hline
\end{tabular}

history was noted in 189 patients (46\%) including - PBD (162), LHM (19), LHM and PBD both (5), and botulinum toxin injection (3).

\section{Procedure characteristics}

An anterior approach ( 2 o'clock) was used in majority of patients (320/408; 78\%), whereas 88 (22\%) patients underwent POEM via posterior approach ( 5 o'clock position). All patients who had previously undergone LHM were treated with posterior myotomy.

The median time of POEM procedure was $76.6 \mathrm{~min}$ ( $30 \mathrm{~min}$ to $180 \mathrm{~min}$ ). Median length of total myotomy was $13 \mathrm{~cm}$ (range $6-21$ ). The median length of myotomy on the esophageal and gastric sides was $10 \mathrm{~cm}$ (range $3-18$ ) and $3 \mathrm{~cm}$ (range $1-4$ ), respectively.

\section{Technical and clinical success}

The technical success rate for the POEM procedure was $97 \%$ $(396 / 408)$ in patients with AC ( $\triangleright$ Table 2$)$. There was no difference in the technical success rates in naïve vs prior treated cases $(97.3 \%$ vs $96.8 \%, P=0.795)$. POEM could not be completed in 12 patients due to excessive submucosal fibrosis ( 9 patients) and inadvertent enlargement of mucosal incision (3 patients). Of the 9 patients with submucosal fibrosis, 4 cases were treatment-naïve and 5 had a history of prior treatment (PBD-3, LHM-2). The majority of patients with submucosal fibrosis underwent PBD (6 patients), whereas 2 patients received botulinum toxin injection and 1 patient with end-stage achalasia underwent esophagectomy. In the 3 patients with inadvertent
- Table 2 Operative findings, adverse events and postoperative follow-up.

Operating time (min) Mean \pm S.D. (Range) $\quad 76.6 \pm 29.1(30-180)$

Site of myotomy

- Anterior

$320(78 \%)$

- Posterior

$88(22 \%)$

Length of myotomy $(\mathrm{cm})$

$13(6-21)$

- Esophageal (cm)

$10(3-18)$

- Gastric (cm)

$3(1-4)$

Adverse events

- Significant mucosal bleeding

0

- Mucosal injury

$18(4.5 \%)$

- Capnothorax

$5(1.2 \%)$

- Capnoperitoneum requiring drainage

$52(13.1 \%)$

- Retroperitoneal air requiring $70(17.6 \%)$ temporary stoppage of procedure

\begin{tabular}{|l|c|}
\hline - Pneumomediastinum & $1(0.2 \%)$ \\
\hline - Pneumopericardium & $1(0.2 \%)$ \\
\hline Other events (not requiring intervention) & \\
\hline - Subcutaneous emphysema & $91(23 \%)$ \\
\hline - Pleural effusion & $1(0.2 \%)$ \\
\hline No. of clips, median (range) & $6(3-22)$ \\
\hline Technical Success & $396 / 408(97 \%)$ \\
\hline Reasons for technical failure & \\
\hline - Submucosal fibrosis & $9(2.2 \%)$ \\
\hline - Mucosal incision enlargement & $3(0.7 \%)$ \\
\hline Hospital stay, mean (range) & $3(2-5$ days) \\
\hline 30 days readmission rate & $1($ Bronchopneu- \\
\hline
\end{tabular}

mucosal incision enlargement, POEM was successfully completed via posterior approach after 4 to 8 weeks.

Of the 396 patients who successfully underwent POEM, 261 (66\%), $172(43.4 \%)$ and 51 (9.6\%) patients completed 1, 2 and 3 years of follow-up, respectively. The median follow-up duration was 17 months (range: $2-42$ months).

The clinical success rates (Eckardt $<4)$ at 1,2 and 3 years were $94 \%(246 / 261), 91 \%(157 / 172)$ and $90.2 \%(46 / 51)$, respectively. At 1 year, the mean Eckardt score significantly decreased from $7.07 \pm 1.6$ at baseline to $1.27 \pm 1.06(P=0.001)$. The clinical success rate in treatment-naïve cases was $95.7 \%$ $(134 / 140)$ as compared to $92.6 \%(112 / 121)$ in previously treated patients at 1 year and was not clinically significant $(P-0.275)$.

In intention to treat analysis (including patients with technical failure and lost to follow up), the clinical success rates were $88.5 \%(246 / 278), 84.4 \%(157 / 186)$ and $46 / 58(79.3 \%)$ at 1,2 and 3 years respectively ( $\bullet$ Table 3 ). 
- Table 3 Number of patients lost to follow-up and those with clinical or technical failure.

\begin{tabular}{|l|c|c|c|}
\hline Follow-up & Clinical failure & Technical ailure & Lost to follow-up \\
\hline 1 year & $15 / 261(5.7 \%)$ & $11 / 278(3.9 \%)$ & $6 / 278(2.1 \%)$ \\
\hline 2 years & $15 / 172(8.7 \%)$ & $9 / 186(4.8 \%)$ & $5 / 186(2.6 \%)$ \\
\hline 3 years & $5 / 51(9.8 \%)$ & $4 / 58(6.8 \%)$ & $3 / 58(5.1 \%)$ \\
\hline
\end{tabular}

- Table 4 Clinical success among Type I, type II and type III achalasia.

\begin{tabular}{|l|l|c|c|c|}
\hline & Type I AC & Type II AC & Type III AC & P value \\
\hline 1 year & $74 / 81(91.3 \%)$ & $158 / 164(96.3 \%)$ & $14 / 16(87.5 \%)$ & $>0.05$ \\
\hline 2 years & $50 / 55(90.9 \%)$ & $100 / 109(91.7 \%)$ & $7 / 8(87.5 \%)$ & $>0.05$ \\
\hline 3 years & $14 / 16(87.5 \%)$ & $29 / 31(93.5 \%)$ & $3 / 4(75.0 \%)$ & $>0.05$ \\
\hline
\end{tabular}

- Table 5 Comparison of objective data before and after POEM procedure.

\begin{tabular}{|l|l|l|l|l|l|}
\hline & Pre-POEM & $\begin{array}{l}\text { Post-POEM } \\
\text { (1 year) }\end{array}$ & $\begin{array}{l}\text { Post-POEM } \\
\text { (2 years) }\end{array}$ & $\begin{array}{l}\text { Post-POEM } \\
\text { (3 years) }\end{array}$ \\
\hline Eckardt score $(\mathrm{n}-408)$ & & $1.27 \pm 1.06$ & $1.12 \pm 0.77$ & $1.2 \pm 0.85$ \\
\hline LES pressure (mean \pm SD) & $7.07 \pm 1.6$ & - & - \\
\hline Integrated relaxation pressure (mean \pm SD) & $26.7 \pm 12.9$ & $9.6 \pm 6.5$ & & $<0.0001$ \\
\hline Timed Barium $(>50 \%$ emptying at 5 minutes) & - & $93.8 \%(213 / 227)$ & - & - \\
\hline LES-Lowe esophageal sphincter & & & $-<0.0001$ \\
\hline
\end{tabular}

There was no significant difference in clinical success among three subtypes of $A C$ (I, II, III) at each follow-up visit (1, 2, 3 years) ( $>$ Table 4 ).

Three patients with clinical failure at one year successfully underwent a re-POEM via alternative route.

\section{Objective data}

Objective data including HRM and TBS were available for 227 out of 261 patients (87\%) who completed 1-year follow-up ( $\triangleright$ Table 5). Mean LES pressure at 1 year after POEM was 15.6 $\pm 6.1 \mathrm{mmHg}$ and significantly less than the pre-procedure LES pressure $(45 \pm 16.5 \mathrm{~mm} \mathrm{Hg})$. Mean integrated relaxation pressures (IRP) before and after POEM were 26.7 \pm 12.9 and $9.6 \pm$ 6.5 , respectively $(P<0.0001)$. Partial restoration of esophageal motility was also observed in previously aperistaltic segments in 6 patients ( $\mathbf{F i g . 6 a , b )}$. These findings were interpreted as ineffective esophageal motility i.e. distal contractile integral $<450 \mathrm{mmHg} \cdot \mathrm{s} \cdot \mathrm{cm}$ in $\geq 50 \%$ swallows as per the Chicago classification of esophageal motility disorders, v3.0.

Of 227 patients, 213 (93.8\%) had more than $50 \%$ reduction in barium column height at 5 minutes ( $\mathbf{F i g . 7 a , b ) . ~}$

\section{Adverse events}

AEs included mucosal perforation, gas-related and other AEs ( $\triangleright$ Table 2). Gas-related events not requiring an intervention, such as drainage or temporary stoppage of procedure, were not considered as AEs. Mucosal perforation was noticed in 18 $(4.5 \%)$ cases. The majority of the perforations (15/18) occurred

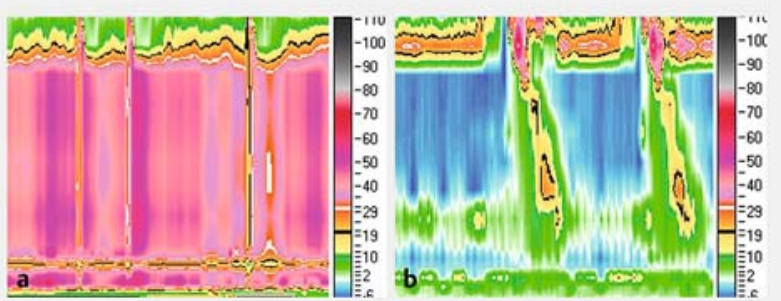

D Fig. 6 a, b High-resolution manometry before POEM and after POEM. Note the appearance of peristaltic wave after POEM ( $\vee$ Fig. 8b).

within $3 \mathrm{~cm}$ of the GEJ. All the perforations were successfully closed by clips and no leak was identified on barium swallow performed on the second postoperative day.

Other events included bleeding and gas-related events. All the intraprocedural bleeding events were controlled with coagrasper (Coagrasper G, FD-412LR, Olympus, Japan) (> Fig.3). There were no major bleeding episodes requiring blood transfusion. None of the patients had delayed bleeding.

The gas-related $A E$ events included capno-thorax in 5 (1.2\%), capno-peritoneum in 52 (13.1\%) and retroperitoneal air in 70 (17.6\%) patients ( $\mathbf{F i g . 8 a , b ) . ~ A l l ~ g a s - r e l a t e d ~ e v e n t s ~ w e r e ~ s u s - ~}$ pected clinically and confirmed by fluoroscopy. Capno-mediastinum and capno-pericardium were noted in 1 patient each. Capno-peritoneum was managed with drainage using conven- 

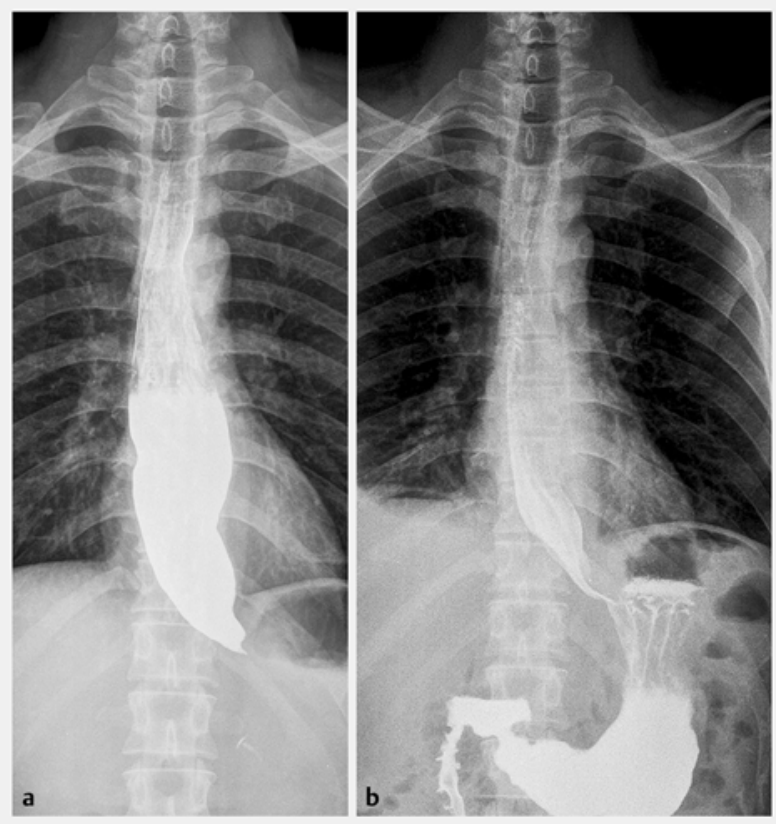

- Fig. 7 a Pre-POEM barium swallow showing complete retention of barium at 5 minutes. b Post-POEM barium swallow showing free flow of barium with complete emptying at 5 minutes.

tional intravenous cannula. Other insufflation-related AEs were managed by temporarily discontinuing the POEM procedure until $\mathrm{CO}_{2}$ was absorbed. Capno-pericardium was incidentally discovered while confirming for the gastric extension of submucosal tunnel. Although it was not associated with hemodynamic compromise, the procedure was withheld for about 15 minutes until $\mathrm{CO}_{2}$ was absorbed.

In addition to the gas-related $\mathrm{AEs}, 1$ patient required readmission within 2 weeks of POEM due to bronchopneumonia which responded to antibiotics.

\section{Other events}

Other events which did not require an intervention included subcutaneous emphysema in 91 (23\%) patients and mild right pleural effusion in 1 patient ( $\mathbf{F i g . 8 c}$ ).

Development of pseudodiverticulae was noted in 5 patients on follow-up endoscopy at 1 year ( Fig.9). However, it was an incidental observation and the clinical significance of same is unknown.

\section{Gastroesophageal reflux disease}

GERD was assessed by clinical symptoms, EGD and pH-impedance analysis ( $\triangleright$ Table 6 ). Clinical symptoms of GERD were detected in 44 out of 261 patients (16.8\%). Twenty-four-hour $\mathrm{pH}$ impedance results were available for 92 patients at 3 months after POEM. A De Meester score of $>14.7$ suggestive of GERD was found in 26 patients (28.3\%).

EGD detected erosive esophagitis in 41 patients $(18.1 \%$; 41/227). Mild (LA grade A-26, LA grade B-11) and severe esophagitis (LA grade C-3 and D-1) was found in 37 and 4 patients respectively.

\section{Discussion}

In this study we demonstrated the safety, efficacy and durability of response to POEM in a large number of subjects with AC with medium-term follow-up.

$A C$ is an incurable disease and the currently available treatments aim at palliation of dysphagia by reducing LES pressures.

The mainstay of endoscopic management in $A C$ is PBD or POEM. The surgical treatment of choice is LHM with partial fundoplication. In the European achalasia trial, LHM and graded PBD were equal in efficacy at 2 and 5 years follow-up. However, a quarter of patients in the PBD group required additional dilatations [5]. Therefore, it appears that the response to PBD is less durable than that for LHM and reintervention requirement is frequent [4].
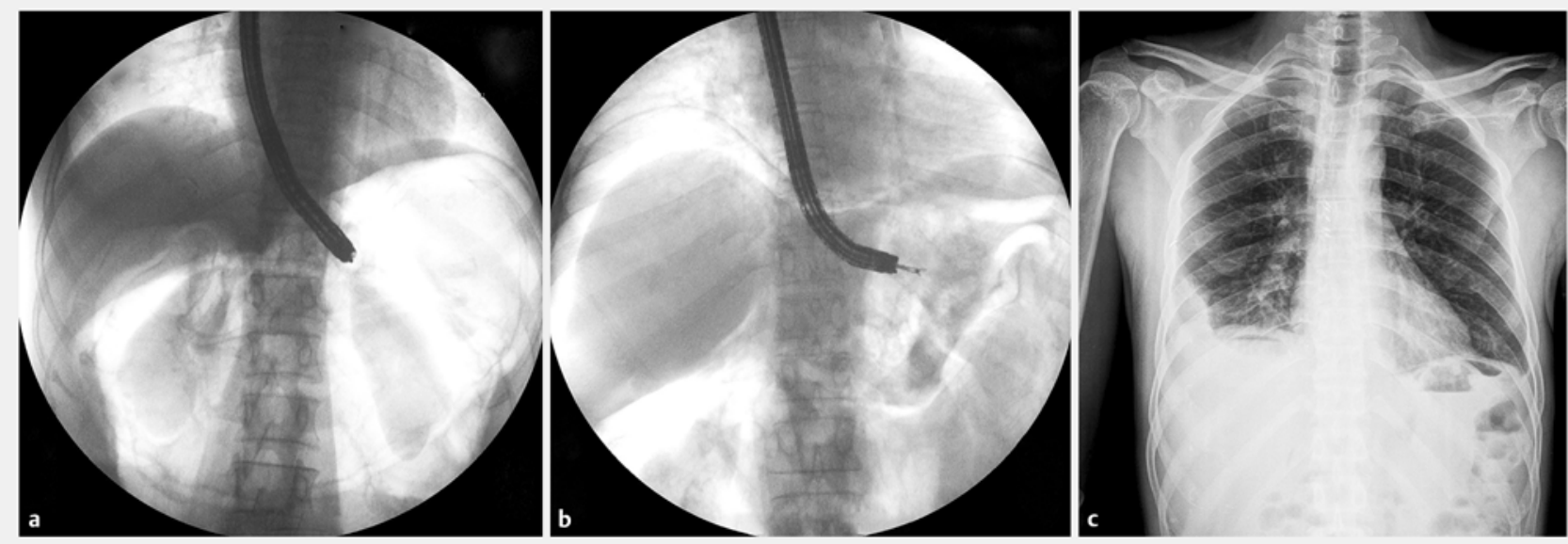

- Fig. 8 Gas-related and other pulmonary adverse events during POEM. a Retroperitoneal gas outlining both kidneys. b Intraperitoneal gas (air under diaphragm). c Mild right-sided pleural effusion. 


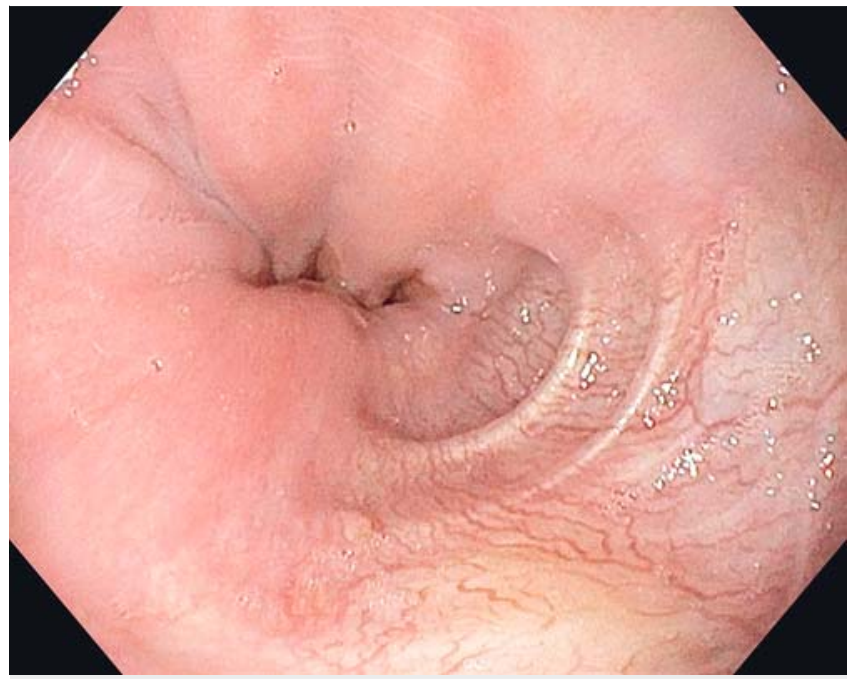

- Fig.9 Pseudo-diverticulum at lower end of the esophagus after posterior POEM in one of the patient.

Table 6 Incidence of GERD after POEM procedure.

\begin{tabular}{|l|c|}
\hline Clinical symptoms (at 1 year follow up) & No. of patients \\
\hline Esophagitis by EGD & $44 / 261(16.8 \%)$ \\
\hline - Grade A/B esophagitis & $41 / 227(18.1 \%)$ \\
\hline - Grade C/D esophagitis & $26 / 11$ \\
\hline De-Meester score > 14.7 & $3 / 1$ \\
\hline GERD, gastroesophageal reflux disease; EGD, esophagogastroduodenoscopy
\end{tabular}

In contrast to LHM, endoscopic myotomy is newer and studies with long-term follow-up are awaited. We have previously reported on short-term outcomes of POEM in over 200 patients with AC. The clinical success rate at 1 year was $92 \%$ and there were no major AEs [9]. Technical and clinical success rates reported in previous studies wer $97 \%$ (95\% Cl: $94-98 \%)$ and $93 \%$ (407/428; $95 \%$ Cl: 90 - $95 \%$ ), respectively [10].

In the current study, we evaluated the efficacy of POEM at longer follow-up periods (i.e. at 1, 2 and 3 years). The clinical success rate at 1 year was $94 \%$, which was maintained when followed up to 3 years ( $90 \%)$. Therefore, POEM is not only effective but the response is also durable. There is paucity of literature regarding the long-term efficacy of POEM. In a series of 500 patients the clinical efficacy rate at 3 years was $88.5 \%$ [11]. In another study, clinical recurrences (later failures) were seen in $17.7 \%$ cases with a total failure rate of $21.5 \%$ at 2 years [12].

Objective testing (TBE and HRM) was available for 227 patients. There was significant reduction in post-POEM LES pressure and height of barium column in TBE. Besides reduction in LES pressures and IRP, partial restoration of esophageal motility was also observed in previously aperistaltic segments in 6 patients ( $\triangleright$ Fig.6a, b). Similar findings have been noted in 1 of

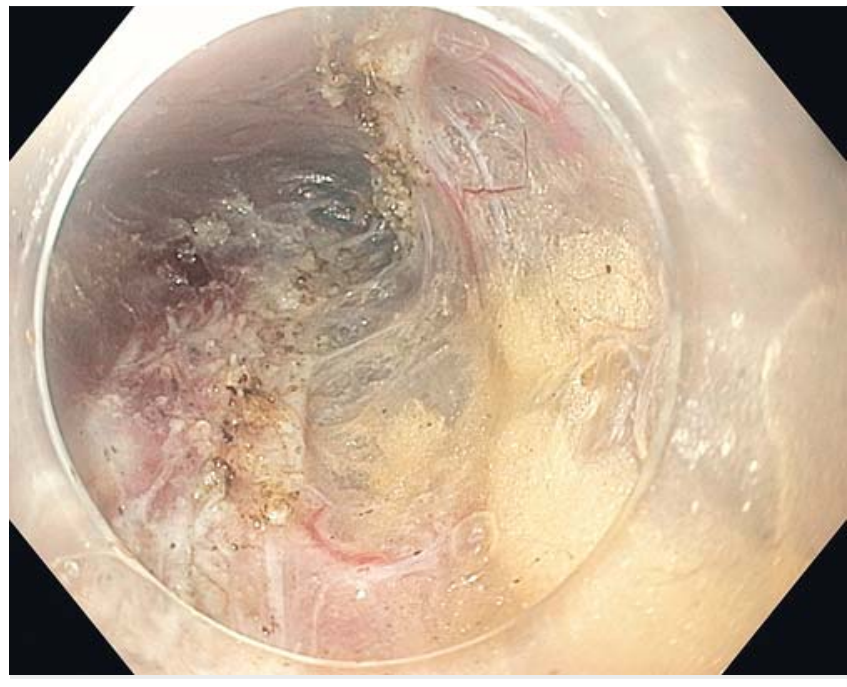

- Fig.10 Glistening peritoneal fat suggesting extension of the submucosal tunnel across the GEJ.

the recently published studies $[13,14]$. However, the clinical impact of the same remains to be seen.

The clinical success of POEM depends to a large extent on the adequacy of myotomy towards the gastric end of the submucosal tunnel [15]. In the initial few cases of learning curve, it may be difficult to correctly identify the GEJ. Moreover, the submucosal tunneling may be prematurely stopped due to the presence of large vessels toward the gastric side of the tunnel. Inadequate extension of the tunnel may lead to early or late recurrences. In a recent study, the outcomes were measured after excluding initial learning curve cases. At an average of 2.4 years post-POEM (range 12 - 52 months), the overall success rate was $92 \%[16]$.

The importance of accurately identifying the GEJ cannot be underestimated. In our study we used retroflexion of scope in the stomach to visualize blanched gastric mucosa, narrowing of tunnel followed by widening of submucosal space, change of vascular pattern and aberrant longitudinal muscle fibers as indicators of GEJ ( $\mathbf{F i g . 4 a - d ) . ~ W h e n ~ i n ~ d o u b t , ~ f l u o r o s c o p y ~}$ was used to ensure passage of the scope across the GEJ [17]. Other methods have been described for identification of the GEJ such as placement of a clip at the GEJ and use of a second endoscope $[18,19]$. In addition to the above methods, we found that visualization of glistening yellow gastric fat during myotomy was a certain indicator of entry into the stomach ( Fig. 10). This has not been described in literature before and needs to be studied further.

The clinical and technical success rates in patients with prior treatment were similar to treatment-naïve cases in our study (92.6\% vs $95.7 \%$ ). The impact of prior treatment such as PBD or LHM on the success of POEM appears to be minimal as found in previous studies [20-22]. However, significant submucosal fibrosis may be encountered in some of these patients, leading to technical difficulty or failure and prolonged procedure duration [23]. In the current study, we found significant submucosal fibrosis in 5 patients who had a history of previous treatment 
with PBD and LHM. In difficult cases with adherent circular muscle fibers, simultaneous myotomy and submucosal dissection can be performed. Care should be taken to avoid loss of planes [24].

Options for failed cases after POEM include PBD, BTI, LHM and re-POEM via an alternate route. In our series the majority of patients in whom POEM failed underwent PBD. Re-POEM was successfully performed in 3 patients with $100 \%$ clinical success at 1-year follow-up. Repeat POEM appears safe and effective as a salvage option after initial POEM failure [25].

The POEM procedure can be performed anteriorly (2 o'clock) or posteriorly (5 o'clock) and largely depends on the operator's preference. In our study, an anterior approach was used in $78 \%$ and a posterior approach in $22 \%$ of patients. A randomized trial is required to compare the safety, efficacy and $A E$ between these 2 approaches.

There were no major AEs related to POEM procedure in our study. Intra-procedural bleeding and gas-related events are part of the procedure, occur frequently and are usually inconsequential [26]. For the same reason, routine chest computed tomography scan is not justified because of the high rate of minor and clinically irrelevant findings [27]. However, caution is advised if tension capno-pericardium develops as cardiac arrest has been reported with this particular gas-related event [28]. In our series, 1 patient developed capno-pericardium which resolved spontaneously after a waiting period of about 15 minutes. It is very important that the complete POEM procedure is carried out under $\mathrm{CO}_{2}$ insufflation, as air takes a much longer time to be absorbed. The utility of underwater POEM to reduce such events remains to be determined [29].

We used endoclips to close all the inadvertent mucoscotomies. Mucosal perforations have been noted in $0 \%$ to $25 \%$ of cases in previous studies [30]. Besides endo-clipping, suturing may also be used to close mucosal defects in difficult cases, although it is more time-consuming than the former [31, 32].

GERD is an important long-term AE after esophageal myotomy. However, there are only a few studies that have objectively determined acid exposure after POEM. In our study abnormal esophageal acid exposure was found in about $28 \%$ of patients. However, clinical symptoms and esophagitis were detected in fewer patients $(17 \%-18 \%)$. The available literature suggests that using a 24-hour $\mathrm{pH}$ study, GERD is present in about $40 \%$ to $50 \%$ of patients after POEM $[16,33,34]$. However, clinically relevant GERD (altered acid exposure with heartburn and/or esophagitis) is found in only one-third to one-quarter of all patients. Therefore, it appears that subjective symptoms are not a reliable indicator of postoperative reflux [35]. The decrease in integrated relaxation pressure after POEM may be a predictor of reflux esophagitis and needs further evaluation [36]. The majority of our patients underwent POEM via an anterior approach, which may theoretically lead to less GERD than a posterior approach. In accordance with our results, symptoms of GERD and reflux esophagitis developed in only about $7 \%$ and $16 \%$ of patients, respectively, after anterior myotomy in another study [36]. However, randomized trials are required to document clinically relevant differences in the occurrence of GERD between these 2 approaches.
The strengths of our study include the large study population, objective evaluation of treatment success and GERD and reasonable duration of follow-up. However, certain drawbacks are noteworthy. The number of patients completing 3-year follow-up was small, pH impedance study could not be performed in all cases and complete GERD evaluation ( $\mathrm{pH}$ study and EGD) was not performed at a single point in time. We may have underestimated the incidence of gas-related events as fluoroscopy was not used in all cases.

\section{Conclusions}

In conclusion, POEM is safe, effective and durable for treatment of AC. The incidence of GERD does not appear to be higher than with LHM. However, randomized comparisons are required. Whether POEM should be offered as a first-line treatment to all patients with $\mathrm{AC}$ is a matter of debate. Long-term follow-up studies and randomized comparison with established modalities such as PBD and LHM will provide conclusive information in that regard.

\section{Competing interests}

None

References

[1] Gockel I, Becker ], Wouters MM et al. Common variants in the HLA-DQ region confer susceptibility to idiopathic achalasia. Nat Genet 2014; 46: $901-904$

[2] Vela MF, Richter JE, Khandwala $F$ et al. The long-term efficacy of pneumatic dilatation and Heller myotomy for the treatment of achalasia. Clin Gastroenterol Hepatol 2006; 4: 580 - 587

[3] Pratap N, Kalapala R, Darisetty S et al. Achalasia cardia subtyping by high-resolution manometry predicts the therapeutic outcome of pneumatic balloon dilatation. J Neurogastroenterol Motil 2011; 17: $48-53$

[4] Yaghoobi M, Mayrand S, Martel M et al. Laparoscopic Heller's myotomy versus pneumatic dilation in the treatment of idiopathic achalasia: a meta-analysis of randomized, controlled trials. Gastrointest Endosc 2013; 78: $468-475$

[5] Moonen A, Annese V, Belmans A et al. Long-term results of the European achalasia trial: a multicentre randomised controlled trial comparing pneumatic dilation versus laparoscopic Heller myotomy. Gut 2016; 65: $732-739$

[6] Swanstrom LL, Kurian A, Dunst CM et al. Long-term outcomes of an endoscopic myotomy for achalasia: the POEM procedure. Ann Surg 2012; 256: 659-667

[7] von Renteln D, Inoue H, Minami H et al. Peroral endoscopic myotomy for the treatment of achalasia: a prospective single center study. The American journal of gastroenterology 2012; 107: 411-417

[8] Swanstrom LL. Poetry is in the air: first multi-institutional results of the per-oral endoscopic myotomy procedure for achalasia. Gastroenterology 2013; 145: 272-273

[9] Ramchandani M, Nageshwar Reddy D, Darisetty S et al. Peroral endoscopic myotomy for achalasia cardia: Treatment analysis and follow up of over 200 consecutive patients at a single center. Digestive 
endoscopy : official journal of the Japan Gastroenterological Endoscopy Society 2016; $28: 19-26$

[10] Barbieri LA, Hassan C, Rosati R et al. Systematic review and meta-analysis: Efficacy and safety of POEM for achalasia. United European gastroenterology journal 2015; 3: 325-334

[11] Inoue H, Sato H, Ikeda H et al. Per-Oral Endoscopic Myotomy: A Series of 500 Patients. J Am Coll Surg 2015; 221: $256-264$

[12] Werner YB, Costamagna G, Swanstrom LL et al. Clinical response to peroral endoscopic myotomy in patients with idiopathic achalasia at a minimum follow-up of 2 years. Gut 2016; 65: 899-906

[13] Hu Y, Li M, Lu B et al. Esophageal motility after peroral endoscopic myotomy for achalasia. Journal of gastroenterology 2016; 51: 458 464

[14] Yao S, Linghu E. Peroral endoscopic myotomy can improve esophageal motility in patients with achalasia from a large sample self-control research (66 patients). PloS one 2015; 10: e0125942

[15] Teitelbaum EN, Sternbach JM, El Khoury R et al. The effect of incremental distal gastric myotomy lengths on EGJ distensibility during POEM for achalasia. Surgl Endosc 2016; 30: 745 - 750

[16] Hungness ES, Sternbach JM, Teitelbaum EN et al. Per-oral Endoscopic Myotomy (POEM) After the Learning Curve: Durable Long-term Results With a Low Complication Rate. Ann Surg 2016; 264: 508 - 517

[17] Kumbhari V, Saxena P, Messallam AA et al. Fluoroscopy to document the extent of cardiomyotomy during peroral endoscopic myotomy. Endoscopy 2014; 46: (Suppl. 01): UCTN: E369-370

[18] Grimes KL, Inoue H, Onimaru M et al. Double-scope per oral endoscopic myotomy (POEM): a prospective randomized controlled trial. Surg Endosc 2016; 30: $1344-1351$

[19] Khashab MA, Kumbhari V, Azola A et al. Intraoperative determination of the adequacy of myotomy length during peroral endoscopic myotomy (POEM): the double-endoscope transillumination for extent confirmation technique (DETECT). Endoscopy 2015; 47: 925 - 928

[20] Sharata A, Kurian AA, Dunst CM et al. Peroral endoscopic myotomy (POEM) is safe and effective in the setting of prior endoscopic intervention. J Gastrointest Surg 2013; 17: 1188-1192

[21] Jones EL, Meara MP, Pittman MR et al. Prior treatment does not influence the performance or early outcome of per-oral endoscopic myotomy for achalasia. Surg Endosc 2016; 30: 1282 - 1286

[22] Fumagalli U, Rosati R, De Pascale $S$ et al. Repeated Surgical or Endoscopic Myotomy for Recurrent Dysphagia in Patients After Previous Myotomy for Achalasia. J Gastrointest Surg 2016; 20: 494-499

[23] Zhou PH, Li QL, Yao LQ et al. Peroral endoscopic remyotomy for failed Heller myotomy: a prospective single-center study. Endoscopy 2013; 45: $161-166$
[24] Li Y, LingHu E, Ding $\mathrm{H}$ et al. Peroral endoscopic myotomy with simultaneous submucosal and muscle dissection for achalasia with severe interlayer adhesions. Gastrointest Endosc 2016; 83: 651 - 652

[25] Li QL, Yao LQ, Xu XY et al. Repeat peroral endoscopic myotomy: a salvage option for persistent/recurrent symptoms. Endoscopy 2016; 48: $134-140$

[26] Yang S, Zeng MS, Zhang ZY et al. Pneumomediastinum and pneumoperitoneum on computed tomography after peroral endoscopic myotomy (POEM): postoperative changes or complications? Acta Radiologica 2015; 56: $1216-1221$

[27] Cai MY, Zhou PH, Yao LQ et al. Thoracic CT after peroral endoscopic myotomy for the treatment of achalasia. Gastrointest Endosc 2014; 80: $1046-1055$

[28] Banks-Venegoni AL, Desilets DJ, Romanelli JR et al. Tension capnopericardium and cardiac arrest as an unexpected adverse event of peroral endoscopic myotomy (with video). Gastrointest Endosc 2015; 82: $1137-1139$

[29] Binmoeller KF, Bhat YM. Underwater peroral endoscopic myotomy. Gastrointest Endosc 2016; 83: 454

[30] Bechara R, Ikeda H, Inoue H. Peroral endoscopic myotomy: an evolving treatment for achalasia. Nature Rev Gastroenterol Hepatol 2015; 12: $410-426$

[31] Pescarus R, Shlomovitz E, Sharata AM et al. Endoscopic suturing versus endoscopic clip closure of the mucosotomy during a per-oral endoscopic myotomy (POEM): a case-control study. Surg Endosc 2016; 30: $2132-2135$

[32] Modayil R, Friedel D, Stavropoulos SN. Endoscopic suture repair of a large mucosal perforation during peroral endoscopic myotomy for treatment of achalasia. Gastrointest Endosc 2014; 80: 1169-1170

[33] Familiari P, Gigante G, Marchese M et al. Peroral Endoscopic Myotomy for Esophageal Achalasia: Outcomes of the First 100 Patients With Short-term Follow-up. Ann Surg 2016; 263: $82-87$

[34] Familiari P, Greco S, Gigante G et al. Gastroesophageal reflux disease after peroral endoscopic myotomy: Analysis of clinical, procedural and functional factors, associated with gastroesophageal reflux disease and esophagitis. Digest Endosc 2016; 28: 33-41

[35] Jones EL, Meara MP, Schwartz JS et al. Gastroesophageal reflux symptoms do not correlate with objective $\mathrm{pH}$ testing after peroral endoscopic myotomy. Surg Endosc 2016; 30: 947 - 952

[36] Shiwaku H, Inoue $\mathrm{H}$, Sasaki T et al. A prospective analysis of GERD after POEM on anterior myotomy. Surg Endosc 2016; 30: 2496 - 2504 